\title{
Indicaciones de angioplastia coronaria después del estudio SYNTAX: ¿Por qué necesitamos nuevos estudios randomizados y nuevas estrategias de revascularización?
}

\author{
Indications for $\mathrm{PCl}$ after the SYNTAX study: ¿Why we need new randomized \\ trials and revascularization strategies?
}

\section{Alfredo E. Rodríguez'}

\begin{abstract}
Resumen
Excluyendo a los pacientes diabéticos, en el pasado los resultados de los estudios aleatorizados que evaluaron la sobrevida no mostraron diferencias entre la cirugía de revascularización miocárdica y la angioplastia coronaria.

En esta revisión se analizan los resultados del seguimiento a corto y largo plazo de un estudio randomizado comparativo entre angioplastia coronaria con implantación de stent farmacológico y cirugía de revascularización miocárdica (Estudio SYNTAX) en pacientes con lesiones coronarias complejas, incluyendo lesiones de tres vasos coronarios y tronco no protegido de coronaria izquierda (TCI). En el texto se discuten los hallazgos, algunos controvertidos e inesperados como el incremento de eventos clínicos adversos "duros" en el grupo angioplastia producto de la mala evolución de los pacientes con lesiones de tres vasos coronarios, que sin embargo no muestra los mismos resultados al comparar los pacientes con lesiones de TCl, en donde la evolución alejada fue similar entre ambos procedimientos de revascularización. En el texto se analizan las posibles causas y eventuales soluciones a estos hallazgos.
\end{abstract}

Palabras clave: angioplastia coronaria, cirugía coronaria, stents, estudios randomizados

\section{INTRODUCCIÓN}

En el pasado, distintos estudios aleatorios que compararon cirugía de revascularización miocárdica (CABG) vs. procedimientos intervencionistas percutáneos (PCI), usando tanto angioplastia coronaria con balón convencional (POBA) como stent metálico (BMS), tuvieron similares tasas de sobrevida e incidencia de infarto de miocardio (IM $)^{1}$ aunque, analizando los resultados de cada uno de estos estudios clínicos, se evidencian algunas diferencias: el bypass Angioplasty Revascularization Investigation (BARI) trial ${ }^{2}$ reportó por primera vez una ventaja de la CABG en la sobrevida de los pacientes diabéticos, ventaja que se mantuvo hasta diez años después de realizado el procedimiento inicial; el Stentor

1. Editor en Jefe de la Revista RACl.

$\bowtie$ Correspondencia: Alfredo E. Rodríguez | arodriguez@centroceci.com.ar | rodrigueza@sanatorio-otamendi.com.ar
Surgery (SoS) trial ${ }^{3}$ presentó mayor mortalidad no cardíaca en todos los pacientes tratados con BMS, y finalmente los resultados del Estudio Randomizado Argentino Angioplastia versus Cirugia (ERACI) mostraron una mejor sobrevida y sobrevida libre de infarto durante los primeros 30 días y durante el primer año en los pacientes tratados con PCI + BMS, ventaja que se mantuvo, aunque no fue significativa, en el seguimiento a 5 años. ${ }^{4,5} \mathrm{~A}$ partir de los estudios mencionados se realizaron varios análisis combinados y metaanálisis, aunque solo en dos de ellos se utilizaron las bases de datos individuales de cada uno de los estudios aleatorios: ${ }^{1,6}$ uno de ellos incluyó a todos los estudios randomizados entre BMS y CABG que evaluaron enfermedad de múltiples vasos, ${ }^{6}$ mientras que en el restante se incluyeron 10 estudios con pacientes tratados tanto con BMS o POBA. ${ }^{1}$

En el primero de los metaanálisis los resultados a cinco años de seguimiento de sobrevida libre de muerte, IM y accidente cerebrovascular (CVA) fue casi idéntica entre BMS y $\mathrm{CABG} ;{ }^{6}$ en este análisis inclusive no se encontró diferencias entre los pacientes diabéticos en el 

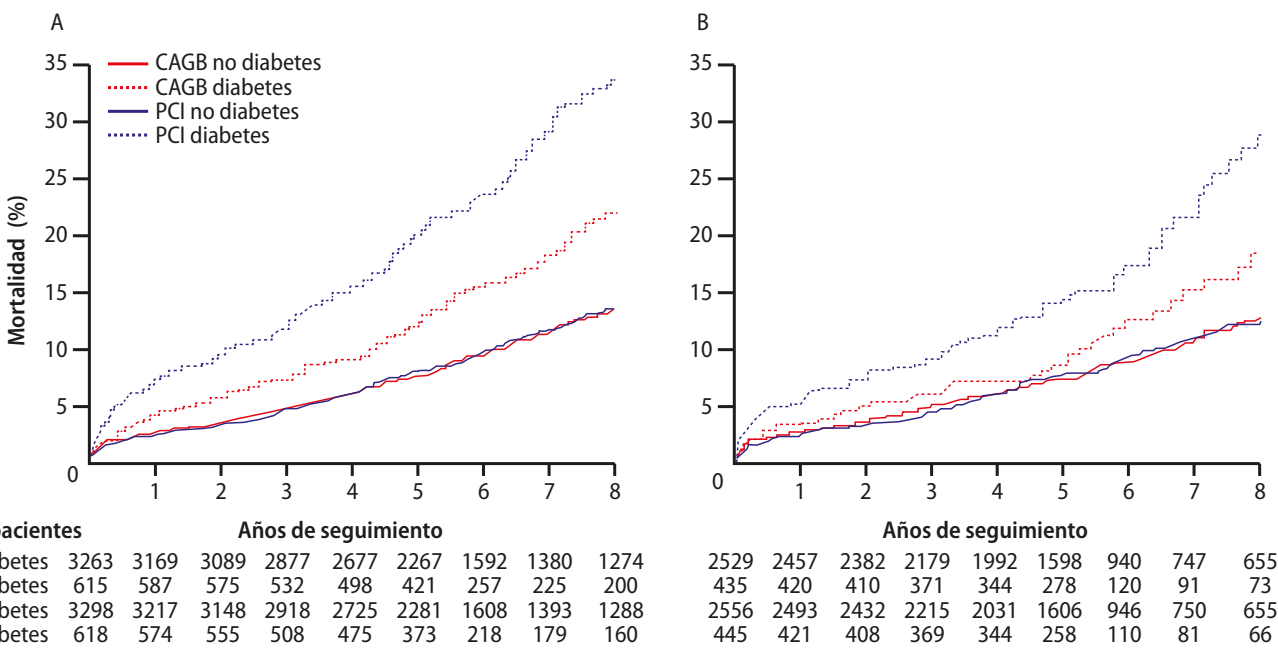

Figura 1. Incidencia de mortalidad en pacientes diabéticos y no diabéticos. A. Incluidos los pacientes del estudio BARI (referencia 2). B. Excluidos los pacientes del estudio BARI (referencia 2). PCI: angioplastia coronaria. CABG: cirugía de revascularización coronaria. Gentileza Dr. Mark Hlatky.

punto combinado de muerte, IM y/o CVA entre ambos grupos terapéuticos. En el estudio de Hlatky hubo similar sobrevida entre los dos procedimientos de revascularización a más de seis años de seguimiento; de hecho, en los pacientes no diabéticos fue casi idéntica aunque los diabéticos tratados con CABG tuvieron una significativa mejor sobrevida (Figura 1) y, en el mismo estudio, los pacientes mayores de 65 años también tuvieron mejor sobrevida con la cirugía de revascularización. En ambos reportes, el número de vasos coronarios involucrados, las lesiones proximales de la arteria descendente anterior y/o la mala función ventricular izquierda no fueron predictores de peor sobrevida con angioplastia coronaria, es decir que la extensión de la enfermedad coronaria no fue un predictor de peor sobrevida con PCI en los estudios aleatorios realizados durante los años 1986/2000. ${ }^{1,6}$ Con la introducción de los primeros diseños de DES en la primera década de este siglo, la reestenosis angiográfica y clínica asociada al uso de BMS disminuyó de forma significativa, y estas ventajas se mantuvieron durante el seguimiento tardío, aparentemente sin aumentar la incidencia de eventos cardiacos "duros" como IM y/o muerte, con lo cual el beneficio para futuras comparaciones con la cirugía coronaria estaba asegurado, ${ }^{7-10}$ inclusive a pesar de que el aumento de la trombosis muy tardía de los primeros diseños de DES y los requerimientos prolongados de la doble terapéutica antiplaquetaria pudieran, eventualmente, disminuir aquellos beneficios. ${ }^{11-14}$

\section{ANÁLISIS DEL ESTUDIO SYNTAX}

A finales de la década pasada se presentaron los resultados a un año de seguimiento del más grande y ambicioso estudio randomizado entre stents liberadores de fármacos vs. CABG en pacientes con lesiones coronarias múltiples llamado The SYNergy between PCI with TAXUS and Cardiac Surgery (SYNTAX) trial. Condu- cido por Serruys, fue un estudio aleatorizado 1:1, multicéntrico y multinacional (85 sitios) que comparó la incidencia de MACE (muerte, IM,CVA y nueva revascularización) entre la primera generación de stents liberadores de fármacos y la cirugía coronaria en pacientes con enfermedad de 3 vasos coronarios y lesiones no protegidas de tronco de coronaria izquierda (TCI).

Los resultados al primer año ${ }^{15}$ mostraron similar incidencia de muerte e IM y una significativa diferencia a favor del grupo DES en la incidencia de CVA $(p=0,003)$, similar tasa de muerte, IM y CVA $(\mathrm{p}=0,89)$ y un mayor requerimiento de nuevas revascularizaciones con DES $(\mathrm{p}=0,001)$, variable que influyó en que el MACE total fuese menor en el grupo CABG $(\mathrm{p}=0,001)$ y por lo cual el grupo PCI no obtuvo márgenes de no inferioridad con respecto a la cirugía.

En este reporte también se estratificaron los resultados de acuerdo con la complejidad de los pacientes mediante un índice, el score de SYNTAX, a mi criterio muy discutido ya que utiliza solo características anatómicas y de procedimiento de angioplastia, que clasificó a la población en estudio en terciles de $<$ de 22 (riesgo leve), entre 22 y 32 (riesgo intermedio) y $>$ de 33 (riesgo alto). Es importante aclarar que el estudio ${ }^{15}$ no tenía poder para evaluar diferencias de acuerdo con este score, por lo cual los resultados de estos subgrupos solo deben ser considerados como generadores de hipótesis para futuros estudios. Además, es solo anatómico y de utilidad para las estrategias de revascularización con angioplastia pero sin ningún efecto en los pacientes sometidos a cirugía coronaria; de hecho, el EuroSCORE en ambos grupos fue similar, solo de 3,8\%, es decir que de acuerdo con este último los pacientes eran de bajo riesgo clínico. ${ }^{15}$

Analizando los resultados de los 1800 pacientes enrolados por el estudio SYNTAX durante el primer año, no se podría ser más optimista: similar incidencia de muerte e IM, incidencia significativamente menor de 
A. Enfermedad de 3 vasos $(n=1095)$

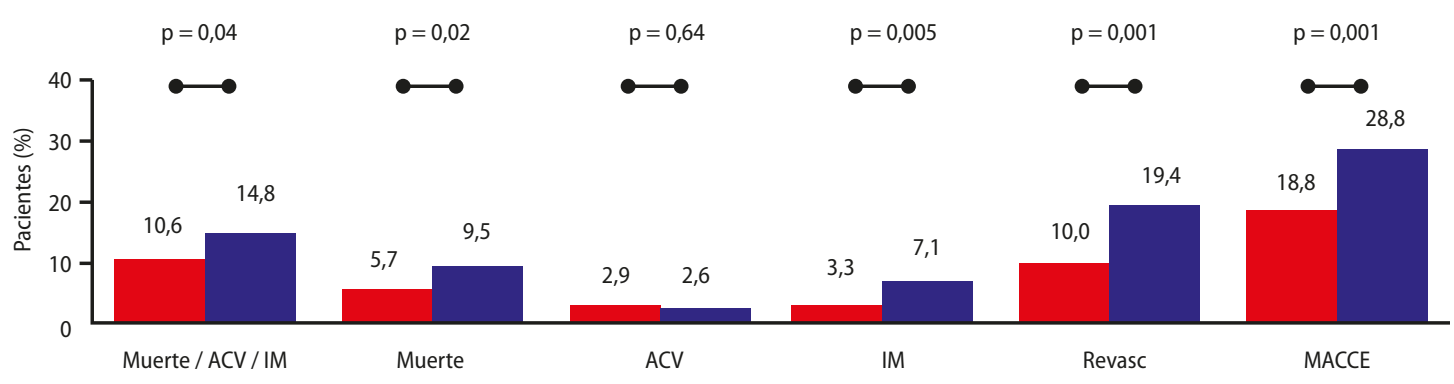

B. Enfermedad de tronco coronario izquierdo $(n=705)$

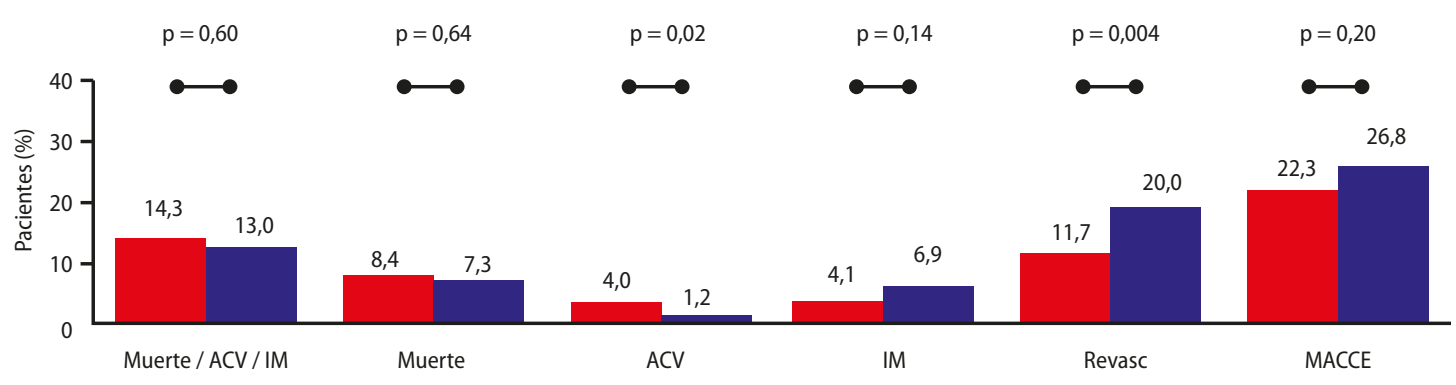

Figura 2. Incidencia de eventos en pacientes tratados con angioplastia en pacientes con lesiones en 3 vasos (A) o tronco coronario izquierdo (B). ACV: accidente cerebrovascular. IM: infarto de miocardio. Revasc: revascularización del vaso tratado. MACCE: eventos adversos cardiacos y cardiovasculares mayores. DES: grupo stent liberador de drogas. CRM: grupo cirugía de revascularización coronaria. Modificado de la referencia 16.

CVA con PCI y, como única ventaja para el grupo cirugía, mayor repetición de revascularización en el grupo DES, hallazgo que se mantuvo invariablemente a favor de CABG en todas las comparaciones aleatorizadas independientemente del device utilizado por la angioplastia: POBA, BMS y ahora DES.

Sin embargo, los seguimientos ulteriores de este estudio a 3 y 4 años mostraron resultados divergentes entre ambas estrategias de revascularización, diferencias que no estaban presentes durante el primer año en varios de los puntos finales.

A tres años de seguimiento en el grupo DES hubo una mayor incidencia de muerte cardíaca $(p=0,02)$, IM $(\mathrm{p}=0,002)$ y nueva revascularización $(\mathrm{p}<0,001)$, mientras que la incidencia de CVA siguió siendo menor en PCI aunque dejando de ser significativa $(p=0,07)$.

Estos hallazgos desfavorables para el grupo angioplastia estuvieron mayormente provocados por la alta incidencia de eventos en los pacientes con lesiones de 3 vasos cuando fueron tratados con angioplastia (Figura $2 \mathrm{~A}$ ); por el contrario, en los pacientes con lesiones de TCI la única ventaja de la cirugía coronaria se debió a una menor tasa de nuevas revascularizaciones. Como vemos en la Figura 2B, la angioplastia presentó una menor incidencia, no significativa, de IM y una menor incidencia, significativa, de CVA $(\mathrm{p}=0,02)$.

A cuatro años de seguimiento, los resultados globales de este estudio mostraron diferencias aún mayores a favor de la rama quirúrgica: muerte global $(p=0,04)$, IM $(\mathrm{p}=0,001)$ y MACE $(\mathrm{p}=0,001)$ siguieron siendo favorables a la cirugía de revascularización. ${ }^{16,17}$
A

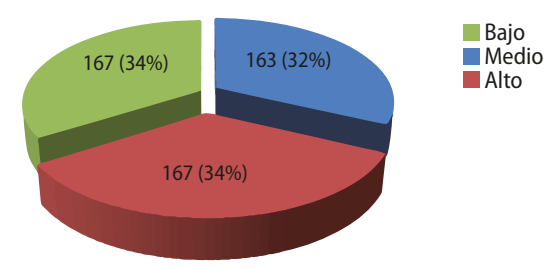

B

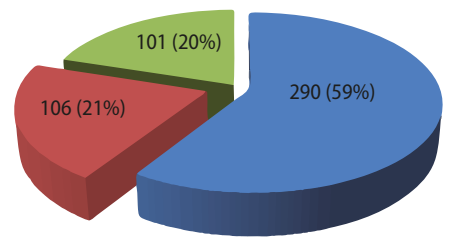

Figura 3. Proporciones de la población del estudio SYNTAX de acuerdo con los terciles clásicos de score SYNTAX $(\boldsymbol{A})$ y luego de revaluación por reserva de flujo coronario (B). Luego de la revaluación, 32\% de los pacientes pasaron de grupos de alto a menor riesgo, tal que 38\% de los terciles altos se movieron a los terciles medios o bajos y $59 \%$ de los medios se movieron al tercil menor; $80 \%$ de los pacientes con score funcional de SYNTAX bajo y/o medio. Modificado referencia 26.

El crecimiento de MACE en el grupo DES después del primer año no solo ocurrió por un aumento de revascularizaciones no planeadas como era de esperar, sino también debido a un incremento, inesperado para muchos de los cardiólogos intervencionistas, de eventos clínicamente duros; entre ellos, infarto de miocardio. La incidencia de IM varió desde 4,8\% en el primer año hasta $8,3 \%$ en el cuarto año para el grupo DES. En contraste, casi no hubo diferencias en la incidencia de IM entre el primero y cuarto año con CABG $(3,3 \%$ y $3,8 \%$, respectivamente). 


\section{¿Cuáles podrían ser los interrogantes y potencia- les respuestas ante estos hallazgos?}

1. ¿Estamos yendo demasiado lejos con las indicaciones de angioplastia?

2. ¿Estamos utilizando el diseño de stent equivocado?

3. ¿Estamos utilizando ahora una estrategia de revascularización inadecuada durante la angioplastia?

La primera pregunta puede ser respondida con los análisis referidos a lo que conocíamos por los estudios previos que compararon angioplastia coronaria y cirugía en la etapa anterior a la introducción de los stents liberadores de fármacos, donde claramente puede verse en la $\mathbf{F i}$ gura 1 que, excluyendo a los pacientes diabéticos, la sobrevida es prácticamente idéntica con ambas estrategias de revascularización. Además, un larguísimo registro recientemente publicado, el estudio ASCERT, observó un hecho inédito: hay un beneficio con la cirugía aun en pacientes con lesiones de 2 arterias coronarias, lo que sugeriría que los beneficios que hoy pueden observarse con CABG van más allá de la extensión de la enfermedad coronaria ${ }^{18} \mathrm{y}$ deben ser explicadas por otras variables.

Con respecto a la segunda pregunta y el uso del stent incorrecto, es cierto que con la primera generación de stents liberadores de fármacos vimos que existe un incremento en los eventos cardíacos adversos después del primer año ${ }^{19-21}$ que puede relacionarse a la incidencia de trombosis muy tardía del stent, que fue del 7,2\% en el estudio SYNTAX. ${ }^{15}$ Esto podría influir en la evolución comparativa con la cirugía coronaria, pudiendo ser una de las explicaciones de los hallazgos a 3 y 4 años del SYNTAX, aunque a mi criterio, no la más relevante.

En los últimos años hemos sido testigos de la introducción de nuevos diseños de DES con nuevas plataformas y polímeros biocompatibles y/o biodegradables que mostraron una sustancial reducción de los eventos cardíacos adversos incluyendo muerte cardíaca e IM en casi todos los subgrupos analizados. Es decir, hoy es posible tratar lesiones muy complejas con resultados clínicos muy favorables comparados con la primera generación de DES, como lo demuestran múltiples estudios aleatorizados. ${ }^{22-24}$ Es más que probable que con estos nuevos diseños una reducción significativa de los eventos adversos en comparación con la cirugía pueda ocurrir en próximos estudios aleatorizados.

Finalmente, la respuesta a la última pregunta, que creo es la más importante para definir: ..."utilizando una estrategia de revascularización inadecuada durante la angioplastia?" Si nosotros analizamos el diseño del estudio SYNTAX obtenemos la respuesta a este interrogante. En el apéndice del trabajo original se indica que el objetivo era la revascularización completa, por lo cual debían revascularizarse arterias de hasta $1,5 \mathrm{~mm}$ de diámetro y lesiones del $50 \%$ y donde se implantaron en promedio más de 4 stents por paciente; $33 \%$ de los casos tuvieron implantados más de $100 \mathrm{~mm}$ de stent y en el $63 \%$ se trataron bifur- caciones, ${ }^{15}$ es decir, es probable que con ese criterio se hubieran tratando con técnicas de bifurcación mucha veces complejas, lesiones intermedias en el vaso principal y ramas secundarias de fino calibre y tamaño; estrategia que es completamente diferente a la utilizada en estudios previos, donde la estrategia de revascularización durante PCI fue lograr una revascularización completa funcional guiada por un análisis anatómico y clínico. ${ }^{4-5,25}$

Esto es más fácil de entender al aplicar una variante funcional del score de SYNTAX como lo han hecho en un trabajo recientemente publicado al utilizar la reserva de flujo coronario para evaluar el grado de severidad de las lesiones coronarias realizando la medición de reserva de flujo para evaluar el score de SYNTAX, y que arrojó que el $66 \%$ de los pacientes con score anatómico de mediano y/o alto grado, al utilizar esta variante de clasificación, fue solo del $41 \%$ y únicamente el $20 \%$ tenía score $>30$, por lo que el valor predictivo de este score funcional fue mucho más selectivo que el "puro" score anatómico del trabajo original; es decir, que la valoración funcional de estos pacientes con lesiones complejas permitió que en el 80\% de los casos estos pacientes presenten anatomía favorable para una angioplastia coronaria con stent ${ }^{26}$ (Figura 3).

\section{CONCLUSIONES E IMPLICANCIAS CLÍNICAS}

Los resultados del estudio SYNTAX nos demuestran que es posible tratar lesiones de tronco de coronaria izquierda con angioplastia con resultados comparables a la cirugía en casos seleccionados. Está claro que para una valoración definitiva serán necesarios nuevos estudios randomizados; hasta hoy todos los estudios aleatorizados no han tenido poder estadístico para demostrarlo, y el estudio EXCEL en curso tendrá el suficiente poder de muestra para lograrlo. ${ }^{27}$ En los pacientes con lesiones de tres vasos coronarios, una estrategia de revascularización funcional, juntamente con nuevos diseños de stents farmacológicos, nos permitirá sin duda seleccionar de forma más racional a los pacientes, y que desde mi punto de vista nos conducirá a una reducción significativa de los eventos adversos de estos pacientes en el seguimiento alejado. Es en este subgrupo, que probablemente represente además el mayor volumen de nuestro trabajo cotidiano, en el que necesitamos con urgencia nuevos estudios aleatorizados comparativos entre ambas estrategias de revascularización.

Hasta que esto no ocurra necesitamos que todos los cardiólogos intervencionistas no olvidemos cuáles fueron nuestros resultados y nuestras estrategias pasadas en este campo en los últimos veinte años.

\section{ABSTRACT}

\section{Indications for PCl after the SYNTAX study: ¿Why we need new randomized trials and revasculari- zation strategies?}

In the past, comparative effectiveness trials evaluating percutaneous coronary interventions (PCI), using either 
balloon angioplasty or bare metal stent (BMS) implantation, versus coronary artery bypass surgery ( $\mathrm{CABG}$ ) found similar survival rates at long-term follow-up with both revascularization strategies. In those reports, number of involved vessels, presence of left anterior descending artery stenosis or poor left ventricular ejection fraction were no predictors of poor survival with PCI. Therefore, extent of the coronary artery disease (CAD) was not associated with poor outcome after PCI in the predrug eluting stent (DES) era. Recently, the SYNTAX (SYNergy between PCI with TAXUS and Cardiac Surgery)a randomized study among drug eluting stents and
CABG in patients with 3 vessels CAD and unprotected left main stenosis (LMS). This study found and increase rate of myocardial infarction and cardiac death beyond the first year of follow up in patients allocated in the DES arm and these findings were driven by poor outcome in patients with 3 vessel CAD whereas no differences death and MI were found in the subgroup of ULMS. The intriguing observation of high mortality rate with $\mathrm{PCI}$ in 3 vessel CAD cohort, meaning a lack of clinical benefit with DES implantation, had not been seen previously. In the manuscript, the author explore for potentials reasons for these results and also searching for potential solutions.

\section{BIBLIOGRAFÍA}

1. Hlatky MA, Boothroyd DB, Bravata DM, et al. Coronary artery bypass surgery compared with percutaneous coronary interventions for multivessel disease: acollaborative analysis of individual patient data from ten randomised trials. Lancet 2009;373:1190-7.

2. The Bypass Angioplasty Revascularization Investigation (BARI) Investigators. Comparison of coronary bypass surgery with angioplasty in patients with multivessel disease. NEngl J Med 1996; 335:217-25. [Erratum, NEngl J Med 1997; 336:147.]

3. Booth J, Clayton T, Pepper J, et al. Randomized, controlled trial of coronary artery bypass surgery versus percutaneous coronary intervention in patients with multivessel coronary artery disease: six year follow-up from the Stent or Surgery Trial (SoS). Circulation 2008;118:381-8.

4. Rodriguez A, Bernardi V, Navia J, et al. Argentine randomized study: Coronary angioplasty with stenting versus coronary bypass surgery in patients with multiple-vessel disease (ERACIII): 30-day and one-year follow-up results. J Am Coll Cardiol 2001;37:51-8.

5. Rodriguez $A E$, Baldi J, Fernández Pereira C, et al. Five-year follow-up of the Argentine randomized trial of coronary angioplasty with stenting versus coronary bypass surgery in patients with multiple vessel disease (ERACI II). J Am Coll Cardiol 2005;46:582-588.

6. Daemen J, Boersma E, Flather M, et al. Long-term safety and efficacy of percutaneous coronary intervention with stenting and coronary artery bypass surgery for multivessel coronary artery disease: a metaanalysis with 5-year patient-level data from the ARTS, ERACI-II, MASS-II, and SOS trials. Circulation 2008; 118:1146-54

7. Moses JW, Leon MB, Popma JJ et al. Sirolimus-eluting stents versus standard stents in patients with stenosis in a native coronary artery. N Engl J Med 2003;349:1315-23.

8. Stone GW, Moses JW, Ellis SG et al. Safety and efficacy of sirolimus-and paclitaxel-eluting coronary stents. N Engl J Med 2007;356:998-1008.

9. Stettler C, Wandel S, Allemann S, et al. Outcomes associated with drugeluting and bare-metal stents: a collaborative network meta-analysis. Lancet 2007;370:937-948.

10. Grube E, Dawkins K, Guagliumi G, et al. TAXUS VI final 5-year results: A multicentre, randomised trial comparing polymer-based moderate-release paclitaxel-eluting stent with a bare metal stent for treatment of long-complex coronary artery lesions. Eurolntervention 2009;4:572-577.

11. Camenzind E, Steg PG, Wijns W. Stent Thrombosis Late After Implantation of First-Generation Drug-Eluting Stents A Cause for Concern. Circulation 2007;115:1440-1455.

12. Lagerqvist B, James SK, Stenestrand U, Lindbäck J, Nilsson T, Wallentin L, for the SCAARStudy Group. Long-term outcomes with drug-eluting stents versus bare-metal stents in Sweden. N Engl J Med 2007;356:1009-1019.

13. Rodriguez AE, Mieres J, Fernandez-Pereira C et al. Coronary stent thrombosis in the current drug-eluting stent era: Insights from the ERACI III trial. J Am Coll Cardiol 2006:47:205-207.

14. Brunner-LaRocca HP, KaiserC, BernheimA, et allnvestigators. Cost-effectiveness of drug-eluting stents in patients athigh orlow risk ofmajorcardiac events in the Basel Stent Kosten Effektivitats Trial (BASKET): an 18-month analysis. Lancet 2007;370:1552-9.

15. Serruys PW, Morice MC, Kappetein AP, et al. Percutaneous coronary intervention versus coronary-artery bypass grafting for severe coronary artery disease. N Engl J Med 2009;360:961-972.

16. Kappetein AP, Feldman TE, MackMJ, et al. Comparison of coronary bypass surgerywith drug-eluting stenting forthetreatment ofleftmain and/orthree-vessel disease:3-yearfollow-up of the SYNTAX trial, 2011. Eur Heart J 2011;32:2125-34.

17. Rodríguez AE, Fernández-Pereira C, Rodríguez-Granillo MA. Changes in the safety paradigm with percutaneous coronary interventions in the modern era: Lessons learned from the ASCERT registry. World J Cardiol 2012 August 26;4(8):242-249.

18. Weintraub WS, Grau-Sepulveda MV, Weiss JM, et al. Comparative effectiveness of revascularization strategies. N Engl J Med 2012. April 19;366(16):1467-76.

19. RodriguezAE, MareeAO, Mieres J, et al. Late loss of early benefit from drugeluting stents when compared with bare-metal stents and coronary artery bypass surgery: 3 years follow-up of the ERACI III registry. Eur Heart J 2007;28:2118-2125.

20. Win HK, Caldera AE, Maresh K, et al. Clinical outcomes and stent thrombosis following off-label use of drug-eluting stents. JAMA 2007;297:2001-9.

21. Rodriguez AE, Rodriguez-Granillo G, Palacios I. Late stent thrombosis: the Damocles sword of drug eluting stents. Eurolntervention 2007 Feb;2(4)512-7.

22. Claessen BE, Stone GW, Smits PC, Kedhi E, Kikkert WJ, Piek JJ, Henriques JP. Would SYNTAX have been a positive trial if XIENCE V have been used instead TAXUS? A meta analysis of a first-generation vs a second generation drug-eluting system Neth Heart J.2010 Sept;18(9):451-53.

23. Caixeta A, Lansky AJ, Serruys PW, et al. Clinical Follow-Up 3 Years After Everolimus- and Paclitaxel-Eluting Stents A Pooled Analysis From the SPIRIT II (A Clinical Evaluation of the XIENCE V Everolimus Eluting Coronary Stent System in the Treatment of Patients With De Novo Native Coronary Artery Lesions) and SPIRIT III (A Clinical Evaluation of the Investigational Device XIENCE V Everolimus Eluting Coronary Stent System [EECSS] in the Treatment of Subjects With De Novo Native Coronary Artery Lesions) Randomized Trials. JACC Cardiovasc Interv 2010 Dec;3(12):1220-8.

24. Claessen BE, Smits PC, Kereiakes DJ, et al. Impact oflesionlength and vessel size on clinical outcomes after percutaneous coronary intervention with everolimus-versus paclitaxel-eluting stents pooled analysis from the SPIRIT (Clinical Evaluation of the XIENCEV Everolimus Eluting Coronary Stent System) and COMPARE (Second-generation everolimus-eluting and paclitaxel-eluting stents in real-life practice) Randomized Trials. JACC Cardiovasc Interv 2011 Nov:4(11):1209-15.

25. Rodriguez AE,Boullon F, Perez Baliño N, et al. Argentine randomized trial of percutaneous transluminal coronary angioplasty versus coronary artery bypass surgery in multivessel disease (ERACI): In-hospital results and 1-year follow-up. J Am CollCardiol 1993;22:1060-1067.

26. Nam CW,Mangiacapra F, Entjes $R$, et al. Functional SYNTAX score for risk assessment in patients with multiple vessel disease. J Am Coll Cardiol 2011;58:1211-1218.

27. Palmerini T, Alessi L and Dangas GRevascularization of unprotected left main coronary disease:Strategy selection and systematic risk assessment. CathCardiovascInterv 2012August 1 ; 80(2):199-205 